\title{
BMJ Open Cannabinoids versus placebo or no intervention for pain: protocol for a systematic review with meta-analysis and trial sequential analysis
}

\author{
Jehad Ahmad Barakji, ${ }^{1}$ Steven Kwasi Korang, ${ }^{1,2}$ Joshua Feinberg, ${ }^{3}$ \\ Mathias Maagard, ${ }^{1}$ Christian Gluud, ${ }^{1}$ Ole Mathiesen, ${ }^{4}$ Janus Christian Jakobsen ${ }^{1,5}$
}

To cite: Barakji JA, Korang SK, Feinberg J, et al. Cannabinoids versus placebo or no intervention for pain: protocol for a systematic review with meta-analysis and trial sequential analysis. BMJ Open 2019;9:e031574. doi:10.1136/ bmjopen-2019-031574

- Prepublication history and additional material for this paper are available online. To view these files, please visit the journal online (http://dx.doi. org/10.1136/bmjopen-2019031574).

Received 11 May 2019 Revised 07 September 2019 Accepted 04 0ctober 2019

Check for updates

(c) Author(s) (or their employer(s)) 2019. Re-use permitted under CC BY-NC. No commercial re-use. See rights and permissions. Published by BMJ.

${ }^{1}$ Copenhagen Trial Unit, Rigshospitalet, Department 7812, Center for Clinical Intervention Research, Copenhagen, Denmark ${ }^{2}$ Pediatric Department, Holbaek Hospital, Holbaek, Denmark

${ }^{3}$ Copenhagen University Hospital, Copenhagen, Denmark ${ }^{4}$ University of Copenhagen, Copenhagen, Denmark

${ }^{5}$ Cardiology Department, Holbaek Hospital, Holbaek, Denmark

Correspondence to Mr Jehad Ahmad Barakji; jehad.barakji@ctu.dk

\section{ABSTRACT}

Introduction Pain is a frequent clinical symptom with significant impact on the patient's well-being. Therefore, adequate pain management is of utmost importance. While cannabinoids have become a more popular alternative to traditional types of pain medication among patients, the quality of evidence supporting the use of cannabinoids has been questioned. The beneficial and harmful effects of cannabinoids in patients with pain is unknown.

Accordingly, we aim to assess the efficacy, tolerability and safety of cannabinoids (herbal, plant-derived extracts and synthetic) compared with placebo or no intervention for any type of pain.

Methods and analyses We will conduct a systematic review of randomised clinical trials with meta-analysis and Trial Sequential Analysis to assess the beneficial and harmful effects of cannabinoids in any dose, formulation and duration. We will accept placebo or no treatment as control interventions. We will include participants with any type of pain (acute and chronic pain, cancer-related pain, headache, neuropathic pain or any other types of pain). We will systematically search The Cochrane Library, MEDLINE, Embase, Science Citation Index and BIOSIS for relevant literature. We will follow the recommendations by Cochrane and the Preferred Reporting Items for Systematic Review and Meta-Analysis statement. The risk of systematic errors (bias) and random errors (play of chance) will be assessed. The overall certainty of evidence will be evaluated using the Grading of Recommendations Assessment, Development and Evaluation approach.

Ethics and dissemination Ethical approval is not a requirement since no primary data will be collected. The findings of this systematic review will be submitted for peer-reviewed publication and disseminated in national and international conferences.

Discussion Although cannabinoids are now being used to manage different pain conditions, the evidence for the clinical effects are unclear. The present review will systematically assess the current evidence for the benefits and harms of cannabinoids to inform practice and future research.

\section{INTRODUCTION}

Pain is the most commonly reported symptom in the general population and in a medical setting. ${ }^{1-3}$ Persistent pain is a major
Strengths and limitations of this study

- Our methodology is based on the Cochrane Handbook for Systematic Reviews of Interventions, the PreferredReporting Items for Systematic Review and Meta-Analysis guideline and a systematic eightstep procedure for valid assessments of statistical and clinical significance.

- We systemically plan to assess risks of random errors ('play of chance') and systematic errors ('bias').

- We have systematically predefined minimal important differences for all outcomes.

- The certainty of the evidence will be assessed using the Grading of Recommendations Assessment, Development and Evaluation approach.

international health problem, ${ }^{4}$ prompting the WHO to endorse a global campaign against pain. ${ }^{5}$ Pain is the leading reason for use of alternative medicines (ie, acupuncture, etc) ${ }^{6}$ Pain has been associated with a low degree of health-related quality of life and may lead to psychosocial distress, insomnia and depressive symptoms. ${ }^{7-15}$ Pain is also among the most common reasons for temporary or permanent work disability. ${ }^{16}$ Pain is always subjective and may be defined as 'an unpleasant sensory and emotional experience associated with actual or potential tissue damage'. ${ }^{17}$

Cannabinoids have emerged as a potential alternative to other painkillers for the treatment of intractable pain. ${ }^{18}$ Before the healthcare systems globally can endorse the applicability of cannabinoids for pain, the potential short-time and long-term benefits and harms with use of cannabinoids must be investigated. This is of utmost importance because patients who consume cannabinoids to alleviate their chronic pain will most likely need to consume cannabinoids for the rest of their lifespan. 


\section{DESCRIPTION OF PAIN}

Pain may be caused by or be related to different clinical disorders and classified according to several different characteristics. ${ }^{19-22}$ Below, we describe shortly some of these classifications.

\section{Acute and chronic pain}

Pain may be classified as 'acute pain' or 'chronic pain'.

- Acute pain usually has a well-defined onset and most often a readily identifiable cause (ie, surgery, etc). Acute pain is expected to run its course in a short time frame and management typically focuses on symptomatic relief until this happens. ${ }^{23}$ Acute pain is a common symptom, affecting between $37 \%$ to $84 \%$ of hospitalised patients. ${ }^{24}$

- Chronic pain is often characterised by an ill-defined onset and a prolonged, fluctuating course. ${ }^{23}$ Chronic pain often persists past normal healing time and hence lacks the acute warning function of physiological nociception. ${ }^{25}$ Pain is usually regarded as chronic when it lasts or recurs for more than 3 to 6 months. ${ }^{1726}$ Chronic pain is a frequent condition, affecting an estimated $20 \%$ of people worldwide ${ }^{27-30}$ and accounting for $15 \%$ to $20 \%$ of physician visits according to European observational studies. ${ }^{31} 32$

\section{Cancer-related pain}

Pain may also be classified based on whether it is cancerrelated or non-cancer-related. Cancer-related pain is pain caused by the cancer itself (primary tumour and metastases) or its treatment (ie, radiation therapy, etc) ${ }^{23} 33$

\section{Postoperative pain}

Postoperative pain includes pain from inflammation caused by tissue trauma (ie, surgical incision, dissection, burns, etc) or direct nerve injury (eg, nerve transection, stretching or compression). ${ }^{34}$ Inflammation results in activation and sensitisation of nociceptive pain pathways, resulting in primary and secondary hyperalgesia and central sensitisation, which is characterised by clinically increased pain, allodynia and increased sensitivity from surrounding non-damaged anatomical areas. ${ }^{35}$

\section{Other types of pain}

Pain in one or more anatomical regions where the aetiology is unknown is defined as idiopathic pain. ${ }^{36}$ Examples of idiopathic pain are chronic widespread pain, fibromyalgia, irritable bowel syndrome and back pain that is not diagnosed as musculoskeletal or as neuropathic pain. ${ }^{33}$

\section{Pain types defined according to specific mechanism causing the pain}

Somatic nociceptive pain

Nociceptive pain is the most frequent type of pain. It results from activity in neural pathways caused by actual tissue damage or potentially tissue-damaging stimuli ${ }^{31} 37$ originating from somatic nociceptors from skin, bone, joints or muscles. $^{38}$
Visceral nociceptive pain

The visceral nociceptive pain is pain resulting from viscera in the thoracic, pelvis or abdominal organs. ${ }^{39-41}$ Visceral pain is diffuse, less distinctive and difficult to localise ${ }^{41}$ and is often characterised by referred visceral pain. ${ }^{42}$

\section{Neuropathic pain}

The 2011 International Association for the Study of Pain definition of neuropathic pain is "pain caused by a lesion or disease of the somatosensory system'. ${ }^{43}$ Neuropathic pain may be classified as central neuropathic pain or peripheral neuropathic pain. Central neuropathic pain conditions are mainly attributed to multiple sclerosis and poststroke pain, ${ }^{44}$ while peripheral neuropathic pain is largely due to postherpetic neuralgia and diabetic neuropathy. ${ }^{45}$

\section{DESCRIPTION OF THE INTERVENTION}

Cannabis (also called marijuana) is the most common illegally used psychoactive substance worldwide. ${ }^{46}$ Cannabinoids refer to a heteromorphic group of molecules that demonstrate activity on cannabinoid receptors. ${ }^{47}$ Cannabinoids may be classified into three groups: (1) endocannabinoids, (2) phytocannabinoids and (3) synthetic cannabinoids. $^{47}$

\section{Endocannabinoids}

Endocannabinoids are characterised by being the endogenously generated cannabinoids. ${ }^{48}$ The primary types of endocannabinoids are the lipid endocannabinoid arachidonoyl ethanolamide (named anandamide) ${ }^{49}$ and the endocannabinoid 2-arachidonoylglycerol. ${ }^{50} 51$

\section{Phytocannabinoids}

Phytocannabinoids are cannabinoids found in the cannabis plant. ${ }^{52}$ The best characterised phytocannabinoids are the psychotropic tetrahydrocannabinol (THC) and the primarily anti-inflammatory cannabidiol. ${ }^{53}$ Nabiximols (marketed as Sativex) is a sublingually administered oromucosal spray based on a mixture of tetrahydrocannabinol and cannabidiol. ${ }^{54}$

\section{Synthetic cannabinoids}

Synthetic cannabinoids are analogues of the cannabinoids found in natural marijuana that are chemically synthesised. The most commonly prescribed cannabinoid-based medicines are the synthetic cannabinoids dronabinol (marketed as Marinol) and nabilone (marketed as Cesamet). ${ }^{54}$

\section{ENDOCANNABINOID SYSTEM}

All cannabinoids act on cannabinoid receptors. These cannabinoid receptors are located throughout the body but are mostly located in the brain. ${ }^{55}$ The cannabinoid receptors and endocannabinoids (see paragraph above) are together named the endocannabinoid system. ${ }^{56}$ 


\section{Cannabinoid receptors}

There are two types of cannabinoid receptors, type I and type II. ${ }^{57}$ Cannabinoid receptor type I are most abundant in the central nervous system, especially in areas promoting nociception, short-term memory and in the basal ganglia, but are also found in the peripheral nerves, uterus, testis and bones. ${ }^{57}$ Tetrahydrocannabinol activates cannabinoid type I receptors in the dopaminergic mesolimbic brain circuit, resulting in enhanced release of dopamine.$^{58}$ Such activation of the so-called 'brain reward system' is hypothesised to mediate the positive reinforcing and rewarding effects of almost all drugs of abuse. ${ }^{58}$

In contrast, cannabinoid receptor type II, is mostly found in the periphery, often in conjunction with immune cells, but may appear in the central nervous system particularly under conditions of inflammation in association with microcytes. ${ }^{57}$ The physiological responses that result from cannabinoid receptor activation are euphoria, psychosis, impaired memory and cognition, reduced locomotor function, increased appetite, as well as anti-emetic, painrelieving, anti-spasticity and sleep-promoting effects. ${ }^{59}$

\section{Administration of cannabinoids}

Cannabis is most commonly consumed via smoked, inhaled vapour or oral routes of administration. ${ }^{60}$ Vaporising cannabis ('vaping') heats the material without burning which theoretically minimises potential carcinogens compared with smoking and may produce less respiratory irritation. ${ }^{61}{ }^{62}$ Sublingual administration is used for some medical cannabis preparations (ie, nabiximols, etc).

\section{WHY IT IS IMPORTANT TO DO THIS REVIEW}

We identified 10 previous reviews with meta-analyses assessing the effects of cannabinoids on different types of pain. ${ }^{63-72}$ Bearing in mind that some of the previous reviews investigated more than one type of pain, eight reviews assessed the effects of different cannabinoids on neuropathic pain ${ }^{63-69} 72$; four reviews assessed the effects of different cannabinoids on nociceptive pain (ie, rheumatoid arthritis, etc) ${ }^{63646768}$; three reviews assessed the effects of different cannabinoids on cancer-related pain ${ }^{636768}$; four reviews assessed the effects of different cannabinoids on fibromyalgia-related pain ${ }^{636467}$ and three reviews assessed the effects of different cannabinoids on postoperative pain. ${ }^{6368} 70$ All the previous reviews included randomised clinical trials, but only 2 of the 10 reviews systematically assessed the risk of bias in the trials, ${ }^{6572}$ and none of the previous reviews took into account the risks of random errors. ${ }^{63-72}$ Only 2 out of the 10 reviews used predefined Cochrane methodology ${ }^{71}$ and only 4 reviews used the Gradingof Recommendations Assessment, Development and Evaluation (GRADE) approach. ${ }^{650-72}$

Most of the reviews concluded that the assessed cannabinoids were effective against pain. ${ }^{63-67} 6972$ In table 1 (online supplementary file 1), we have summarised the results and conclusions of the previous reviews. Five of the reviews reported serious adverse events (ie, agitation, impaired memory, abuse, dissociation, acute psychosis, death, etc).$^{63-6772}$ The reviews also showed that the most commonly reported adverse events were sedation, dizziness, dry mouth, increased appetite, somnolence, confusion, nausea and disturbances in concentration. ${ }^{63-66} 68697172$

A correlation between psychiatric disorders (schizophrenia, psychosis, etc) and increased cannabinoid consumption has previously been hypothesised. ${ }^{73-79}$ Di Forti et al recently conducted a study indicating that daily cannabis use was associated with increased odds of psychotic disorders compared with never users (adjusted OR $3.2,95 \%$ CI 2.2 to 4.1 ), increasing to nearly five-times increased odds for daily use of high-potency (THC $\geq 10 \%$ ) types of cannabis (adjusted OR $4.8,95 \%$ CI 2.5 to 6.3 ).$^{80}$

Compared with previous systematic reviews on cannabinoids, we want to assess the effects of all types of cannabinoid versus placebo or no intervention for all different forms of pain. Depending on the data results provided by the included trials this could increase the power and precision of the overall analysis and make it possible to conduct subgroup analyses and sensitivity analyses that may identify pain areas where cannabinoid could be especially beneficial and cause the least harms. In addition, we will implement a minimal clinically important threshold regarding analgesic efficacy based on previously conducted methodological studies which ensures that analgesic efficacy is of a firm significance before acceptance. Finally, by instigating all types of cannabinoids treated for any type of pain this systematic review will aid trialist in optimising the design of future randomised clinical trials by illuminating any research pitfalls of all previously conducted randomised clinical trials on this topic.

\section{Objective}

The objective of our systematic review is to assess the analgesic efficacy and adverse events encumbered with the use of cannabinoids compared with placebo or no intervention in participants with any type of pain (acute and chronic pain, cancer-related pain, headache, neuropathic pain or any other types of pain). A secondary objective of this systematic review is to assess the impact of cannabinoid use on the quality of sleep and quality of life which is especially decreased in participants with chronic pain.

\section{METHODS}

This systematic review protocol has been developed based on Preferred Reporting Items for Systematic Reviews and Meta-Analysis Protocols (PRISMA-P) guidelines for reporting systematic reviews evaluating healthcare interventions. ${ }^{8182}$ A PRISMA-P checklist file is attached (online supplementary file 2 ).

\section{CRITERIA FOR CONSIDERING STUDIES FOR THIS REVIEW Type of studies}

Randomised clinical trials irrespective of trial design, setting, publication status, publication year and language. 
If we identify quasi-randomised studies and observational studies during our searches for randomised clinical trials, we will only include their reporting on harms in a narrative way. By not systematically searching for all observational studies on harm, we run the risk of putting more focus on benefit than harm. We are aware that this is a limitation of our review.

\section{Types of participants}

Participants with any type of pain, that is, acute and chronic pain, cancer-related pain, headache, neuropathic pain or any other types of pain (as defined by the trialists). Participants will be included irrespective of age, sex and comorbidities.

\section{Types of interventions \\ Experimental intervention}

Any type of cannabinoids such as: herbal cannabis (hashish, marijuana), plant-based extracts (ie, nabiximols, etc) or synthetic cannabinoids (ie, cannabidiol, dronabinol, levonantradol, nabilone, etc). We will accept cannabinoids at any dose, by any route, administered for the relief of pain.

Control intervention

Placebo or no intervention.

\section{Co-interventions}

We will accept any co-intervention but only if this co-intervention is planned to be delivered similarly in both intervention groups. If this plan is not followed, then these trials will be assessed as a subgroup due to potential confounding.

\section{Types of outcome measures}

Primary outcomes

- All-cause mortality.

- Pain assessment on Visual Analogue Scale (VAS) or Numerical Rating Scale (NRS).

- Proportion of participants with a serious adverse event defined as any untoward medical occurrence that resulted in death; was life threatening; was persistent or led to significant disability, nephrotoxicity, superinfection, need for respiratory support, need for circulatory support or prolonged hospitalisation. ${ }^{83}$ As we expect the trialists' reporting of serious adverse events to be heterogeneous and not strictly according to the International Conference on Harmonisation - Good Clinical Practice (ICH-GCP) recommendations, we will include the event as a serious adverse if the trialists either: (1) use the term 'serious adverse event' but not refer to ICH-GCP, or (2) report the proportion of participants with an event we consider fulfils the ICHGCP definition. If several of such events are reported then we will choose the highest proportion reported in each trial.

- Quality of life measured on any valid continuous scale.

Secondary outcomes

- Dependence (as defined by trialists).
- Psychosis (as defined by trialists).

- Proportion of participants with one or more adverse event not considered to be serious.

- Quality of sleep measured on any valid continuous scale.

\section{Exploratory outcomes}

- Each serious adverse event separately.

- Each adverse event not considered serious separately.

- Twenty-four hour morphine consumption (as defined by trialists).

- Physical function (as defined by trialists).

- Depressive symptoms (eg, Hamilton Depression Rating Scale).

We will for all outcomes use the trial results reported at maximal follow-up except for acute pain. For acute pain, we will use the trials' results reported at the time point closest to 24 hours after the intervention is given.

\section{Patient and public involvement}

We have had email correspondence with several relevant patient associations in Denmark to select the most patientrelevant outcomes. The patient associations we have been in contact with included: The Danish Diabetes Association, Steno Diabetes Centre Copenhagen, The Danish Rheumatism Association, The Danish Multiple Sclerosis Society and Danish Cancer Society. Initially we presented our potential outcomes for the patient associations and requested for their opinion. We had not included quality of sleep as an outcome, however, this was mentioned by almost all patient associations and it was included as a crucial secondary outcome. All-cause mortality was questioned by one of the patient associations, however, we want to keep this outcome because of potential increased risk of both acute coronary syndrome and chronic cardiovascular disease associated with cannabis use. ${ }^{84}$

\section{Search methods for identification of studies \\ Electronic searches}

We will search the Cochrane Central Register of Controlled Trials (CENTRAL), Medical Literature Analysis and Retrieval System Online (MEDLINE), Excerpta Medica database (Embase), Latin American and Caribbean Health Sciences Literature (LILACS), Science Citation Index Expanded on Web of Science and BIOSIS in order to identify relevant trials. The preliminary search strategy for CENTRAL, MEDLINE (Ovid), Embase (Ovid), LILACS, Web of Science and BIOSIS is given in online supplementary file 3 .

We will search all databases from their inception to the $1^{\text {st }}$ of October 2019.

\section{Searching other resources}

The reference lists of relevant publications will be checked for any unidentified randomised trials. We will contact authors of included studies, and major pharmaceutical companies, by email asking for unpublished randomised trials. Further, we will search for ongoing trials on:

- ClinicalTrials.gov (www.clinicaltrials.gov) 
- Google Scholar (https://scholar.google.dk/)

- The Turning Research into Practice (TRIP) Database (https://www.tripdatabase.com/)

- European Medicines Agency (EMA) (http:// www. ema.europa.eu/ema/)

- United States Food and Drug Administration (FDA) ( www.fda.gov)

- China Food and Drug Administration (http://eng. sfda.gov.cn/WS03/CL0755/)

- Medicines and Healthcare products Regulatory Agency (https://www.gov.uk/government/organisations / medicines-and-healthcare-products-regulatoryagency)

- The WHO International Clinical Trials Registry Platform search portal (http://apps.who.int/ trialsearch/)

We will also consider relevant for the review unpublished and grey literature trials, if we identify such trials.

\section{Data collection and analysis}

We will perform the review following the recommendations of Cochrane. ${ }^{85}$ The analyses will be performed using Review Manager $5^{86}$ and Trial Sequential Analysis. ${ }^{87}$ In case of Review Manager statistical software is not being sufficient, we will use Stata $15 .^{88}$

\section{Selection of studies}

Two authors (JB, SKK) will independently screen titles and abstracts. We will retrieve all relevant full-text study reports/publications, and four review authors (JB, SKK, JRF, MM) will independently screen the full texts and identify and record reasons for exclusion of the ineligible studies. We will resolve any disagreement through discussion or, if required, we will consult a fifth author (JCJ). Trial selection will be displayed in an adapted flow diagram as per the Preferred Reporting Items for Systematic Reviews and Meta-Analyses statement. ${ }^{89}$

\section{Data extraction and management}

Four authors (JB, SKK, JRF, MM) will in pairs extract data independently from included trials. Disagreements will be resolved by discussion with a fifth author (JCJ). We will assess duplicate publications and companion papers of a trial together to evaluate all available data simultaneously (maximise data extraction, correct bias assessment). We will contact the trial authors by email to specify any additional data, which may not have been reported sufficiently or at all in the publication.

\section{Trial characteristics}

Bias risk components (as defined below), trial design (parallel, factorial or crossover), number of intervention arms, length of follow-up, estimation of sample size, inclusion and exclusion criteria.

\section{Participant characteristics and diagnosis}

Number of randomised participants, number of analysed participants, number of participants lost to follow-up/ withdrawals/crossover, compliance with medication, age range (mean or median) and sex ratio, type of pain (acute and chronic pain, cancer-related pain, headache, neuropathic pain or any other types of pain), baseline pain score, drug and dosing regimen, study design (placebo or active control), study duration and follow-up, analgesic outcome measures and results, adverse events (participants experiencing any adverse event, or serious adverse event).

\section{Co-intervention characteristics}

Type of co-intervention, dose of co-intervention, duration of co-intervention and mode of administration.

\section{Outcomes}

All outcomes listed above will be extracted from each randomised clinical trial, and we will identify if outcomes are incomplete or selectively reported according to the criteria described later in 'incomplete outcome data' bias domain and 'selective outcome reporting' bias domain.

Notes

Funding of the trial and notable conflicts of interest of trial authors will be extracted, if available.

We will note in the 'Characteristics of included studies' table if outcome data were not reported in a usable way. Four review authors (JB, SKK, JRF, MM) will independently transfer data into the Review Manager file. ${ }^{86}$ Disagreements will be resolved through discussion or, if required, we will consult with a fifth author (JCJ).

\section{Assessment of risk of bias in included studies}

We will use the instructions given in the Cochrane Handbook for Systematic Reviews of Interventions ${ }^{85}$ in our evaluation of the methodology and hence the risk of bias of the included trials. We will evaluate the methodology in respect of:

- Random sequence generation.

- Allocation concealment.

- Blinding of participants and treatment providers.

- Blinding of outcome assessment.

- Incomplete outcome data.

- Selective outcome reporting.

- For-profit bias.

- Overall risk of bias.

These components enable classification of randomised trials as being at low risk of bias and at high risk of bias. The latter trials tend to overestimate positive intervention effects and underestimate negative effects. ${ }^{90-96}$

We will classify the trials according to the following criteria.

\section{Random sequence generation}

- Low risk: If sequence generation was achieved using computer random number generator or a random number table. Drawing lots, tossing a coin, shuffling cards and throwing dice were also considered adequate if performed by an independent adjudicator.

- Unclear risk: If the method of randomisation was not specified, but the trial was still presented as being randomised. 
- High risk: If the method of sequence generation was inadequate for example, alternate medical record numbers or other non-random sequence generation.

\section{Allocation concealment}

- Low risk: If the allocation of patients was performed by a central independent unit, on-site locked computer, identical-looking numbered sealed envelopes, drug bottles or containers prepared by an independent pharmacist or investigator.

- Uncertain risk: If the trial was classified as randomised but the allocation concealment process was not described.

- High risk: If the allocation sequence was familiar to the investigators who assigned participants.

\section{Blinding of participants and treatment providers}

- Low risk: If the participants and the treatment providers were blinded to intervention allocation and this was described.

- Uncertain risk: If the procedure of blinding was insufficiently described.

- High risk: If blinding of participants and the treatment providers was not performed.

\section{Blinding of outcome assessment}

- Low risk of bias: If it was mentioned that outcome assessors were blinded and this was described.

- Uncertain risk of bias: If it was not mentioned if the outcome assessors in the trial were blinded or the extent of blinding was insufficiently described.

- High risk of bias: If no blinding or incomplete blinding of outcome assessors was performed.

\section{Incomplete outcome data}

- Low risk of bias: If missing data were unlikely to make treatment effects depart from plausible values. This could be either (1) there were no dropouts or withdrawals for all outcomes or (2) the numbers and reasons for the withdrawals and dropouts for all outcomes were clearly stated and could be described as being similar to both groups. Generally, the trial is judged as at a low risk of bias due to incomplete outcome data if dropouts are less than $5 \%$. However, the $5 \%$ cut-off is not definitive.

- Uncertain risk of bias: If there was insufficient information to assess whether missing data were likely to induce bias on the results.

- High risk of bias: If the results were likely to be biassed due to missing data either because the pattern of dropouts could be described as being different in the two intervention groups or the trial used improper methods in dealing with the missing data (ie, last observation carried forward, etc).

Selective outcome reporting

- Low risk of bias: If a protocol was published before or at the time the trial was begun, and the outcomes specified in the protocol were reported on. If there is no protocol or the protocol was published after the trial has begun, reporting pain assessment on VAS or NRS and serious adverse events will grant the trial a grade of low risk of bias.

- Uncertain risk of bias: If no protocol was published and the outcome pain assessment on VAS or NRS and serious adverse events were not reported on.

- High risk of bias: If the outcomes in the protocol were not reported on.

\section{For-profit bias}

- Low risk of bias: If the trial appeared to be free of other components of for-profit bias.

- Unclear risk of bias: If it was unclear whether the trial was free of for-profit bias.

- High risk of bias: If there was a high risk of for-profit bias.

\section{Overall risk of bias}

- Low risk of bias: The trial will be classified at overall 'low risk of bias' only if all of the bias domains described in the above paragraphs are classified at 'low risk of bias'.

- High risk of bias: The trial will be classified at 'high risk of bias' if any of the bias risk domains described in the above are classified at 'unclear' or 'high risk of bias'.

We will assess the domains 'blinding of outcome assessment', 'incomplete outcome data' and 'selective outcome reporting' for each outcome result. Thus, we can assess the bias risk for each outcome assessed in addition to each trial. Our primary conclusions will be based on the results of our primary outcome results at overall low risk of bias. Both our primary and secondary analyses will be presented in the summary of findings tables.

\section{Differences between the protocol and the review}

We will conduct the review according to this published protocol and report any deviations from it in the 'Differences between the protocol and the review' section of the systematic review.

\section{Measures of treatment effect \\ Dichotomous outcomes}

We will calculate risk ratios with $95 \%$ CI for dichotomous outcomes, as well as the Trial Sequential Analysis-adjusted CIs (see below).

\section{Continuous outcomes}

We will calculate the mean differences and the standardised mean difference with 95\% CI for continuous outcomes, as well as the Trial Sequential Analysis-adjusted CIs (see below).

\section{Dealing with missing data}

We will, as first option, contact all trial authors to obtain any relevant missing data (ie, for data extraction and for assessment of risk of bias, as specified above). 


\section{Dichotomous outcomes}

We will not impute missing values for any outcomes in our primary analysis. In two of our sensitivity analyses (see paragraph below), we will impute data.

\section{Continuous outcomes}

We will primarily analyse scores assessed at single time points. If only changes from baseline scores are reported, we will analyse the results together with follow-up scores. ${ }^{85}$ If SDs are not reported, we will calculate the SDs using trial data, if possible. We will not use intention-to-treat data if the original report did not contain such data. We will not impute missing values for any outcomes in our primary analysis. In our sensitivity analysis (see paragraph below) for continuous outcomes, we will impute data.

\section{Assessment of heterogeneity}

We will primarily investigate forest plots to visually assess any sign of heterogeneity. We will secondly assess the presence of statistical heterogeneity by $\mathrm{X}^{2}$ test (threshold $\mathrm{p}<0.10$ ) and measure the quantities of heterogeneity by the $\mathrm{I}^{2}$ statistic. ${ }^{9798}$ We will investigate for heterogeneity through subgroup analyses. Ultimately, we may decide that a meta-analysis should be avoided. ${ }^{85}$

\section{Assessment of reporting biases}

We will use a funnel plot to assess reporting bias if 10 or more trials are included. We will visually inspect funnel plots to assess the risk of bias. We are aware of the limitations of a funnel plot (ie, a funnel plot assesses bias due to small sample size, etc). From this information, we assess possible reporting bias. For dichotomous outcomes, we will test asymmetry with the Harbord test ${ }^{99}$ if $\tau^{2}$ is less than 0.1 and with the Rücker test if $\tau^{2}$ is more than 0.1 . For continuous outcomes, we will use the regression asymmetry test ${ }^{100}$ and the adjusted rank correlation. ${ }^{101}$

\section{Unit of analysis issues}

We will only include randomised clinical trials. For trials using crossover design, only data from the first period will be included. ${ }^{85102}$ There will therefore not be any unit of analysis issues.

\section{Minimal important difference}

In clinical intervention research it is of utmost importance always to define minimal important differences and to define thresholds for clinical significance. ${ }^{103}$ If a large number of trial participants are randomised, small and clinically irrelevant intervention effects may lead to statistically significant results and rejection of the null hypothesis. ${ }^{104}$ Jaeschke et al defined the minimal important difference as 'the smallest difference in score in the domain of interest which patients perceive as beneficial'. ${ }^{105}$

Estimations of minimal important differences should be used as arbitrary strict precise thresholds. However, to avoid erroneous conclusions minimal important differences need to be estimated and predefined when assessing the effects of interventions for pain. Olsen et al have conducted two systematic reviews on this matter in order to gather the evidence and present an estimate of the minimal important difference. ${ }^{106107}$ Olsen et al conducted a systematic review on the minimal important difference in patients with acute pain and concluded that the median of the studies' results was $17 \mathrm{~mm}$ on VAS (IQR $14 \mathrm{~mm}$ to $23 \mathrm{~mm}$ ). ${ }^{106}$ Another systematic review conducted by Olsen et al was on the minimal important difference in patients with chronic pain and the results showed a median of $23 \mathrm{~mm}$ on VAS (IQR 12 $\mathrm{mm}$ to $39 \mathrm{~mm}$ ) when using the within-patient anchor-based method, while the median in studies using the sensitivitybased and specificity-based method was $20 \mathrm{~mm}$ on VAS (IQR $15 \mathrm{~mm}$ to $30 \mathrm{~mm}$ ). ${ }^{107}$ We have described detailed considerations about minimal important differences in online supplementary appendix 4.

Based on the previously conducted systematic reviews we will choose at minimal important difference equivalent to $10 \mathrm{~mm}$ or 1 point on the visual analogue scale and the numerical rating scale, respectively, regarding an analgesic effect.

\section{Data synthesis}

Meta-analysis

We will undertake this meta-analysis according to the recommendations stated in the Cochrane Handbook for Systematic Reviews of Interventions, ${ }^{85}$ Keus et al ${ }^{108}$ and the eight-step assessment suggested by Jakobsen et $a l .{ }^{103}$ We will use the statistical software Review Manager $5.3^{86}$ provided by Cochrane to analyse data. We will assess our intervention effects with both random-effects metaanalyses ${ }^{109}$ and fixed-effect meta-analyses. ${ }^{110}$ We will use the more conservative point estimate of the two. ${ }^{103}$ The more conservative point estimate is the estimate closest to zero effect. If the two estimates are similar, we will use the estimate with the highest $\mathrm{p}$ value. ${ }^{103}$ We use four primary and four secondary outcomes, and therefore, we will consider a $\mathrm{p}$ value of 0.02 as the threshold for statistical significance. ${ }^{103111}$ We will investigate for heterogeneity through subgroup analyses. Ultimately, we may decide that a meta-analysis should be avoided. ${ }^{85}$ We will use the eight-step procedure to assess if the thresholds for statistical and clinical significance are crossed. ${ }^{103}$ Our primary conclusion will be based on results with low risk of bias. ${ }^{103}$

Where multiple trial intervention groups are reported in a single trial, we will include only the relevant groups. If two comparisons are combined in the same metaanalysis, we will halve the control group to avoid double counting. ${ }^{85}$ Trials with a factorial design will be included.

If quantitative synthesis is not appropriate, we will report the results in a narrative way.

\section{Trial sequential analysis}

Traditional meta-analysis runs the risk of random errors due to sparse data and repetitive testing of accumulating data when updating reviews. We wish to control the risks of type I errors and type II errors. We will therefore perform Trial Sequential Analysis on the outcomes, in order to calculate the required information size (that is the number of participants needed in a meta-analysis 
to detect or reject a certain intervention effect) and the cumulative Z-curve's breach of relevant trial sequential monitoring boundaries. ${ }^{87112-120}$ A more detailed description of Trial Sequential Analysis can be found in the Trial Sequential Analysis manual ${ }^{113}$ and at http://www.ctu.dk/ tsa/. For dichotomous outcomes, we will estimate the required information size based on the observed proportion of patients with an outcome in the control group (the cumulative proportion of patients with an event in the control groups relative to all patients in the control groups), a relative risk reduction of $20 \%$, an alpha of $2.0 \%$ for our primary and secondary outcomes, a beta of $10 \%$ and diversity as suggested by the trials in the metaanalysis. For the outcome "pain assessment on visual analogue scale or numerical rating scale', we will use a minimal important difference estimate based on previously conducted systematic reviews. ${ }^{106} 107$ We will accept an analgesic effect equivalent to $10 \mathrm{~mm}$ or 1 point on the visual analogue scale and the numerical rating scale, respectively, or a consumption of at least $5 \mathrm{mg}$ morphine.

For all remaining continuous outcome, we will in the Trial Sequential Analysis use the observed SD, a mean difference of the observed $\mathrm{SD} / 2$, an alpha of $2.0 \%$ for our primary and secondary outcomes and a beta of $10 \%$.

\section{Subgroup analysis and investigation of heterogeneity Subgroup analysis}

We will perform the following subgroup analysis when analysing the primary outcomes (all-cause mortality, pain assessment on VAS or NRS, serious adverse event and quality of life).

- Trials at high risk of bias compared with trials at low risk of bias.

- Trials at risk of vested interests compared with trial with no risk of vested interests.

- Trials compared according to type of pain (acute pain, chronic pain and cancer pain).

- Trials compared according to type of chronic pain.

- Trials compared according to type of cannabinoids used.

We will use the formal test for subgroup interactions in Review Manager. ${ }^{86}$

\section{Sensitivity analysis}

To assess the potential impact of the missing data for dichotomous outcomes, we will perform the two following sensitivity analyses on both the primary and secondary outcomes.

- 'Best-worst-case' scenario: We will assume that all participants lost to follow-up in the cannabinoid intervention group have survived, and had no serious adverse event, and that all those participants lost to follow-up in the placebo group have not survived, and had a serious adverse event.

- 'Worst-best-case' scenario: We will assume that all participants lost to follow-up in the cannabinoid intervention group have not survived, and had a serious adverse event, and that all those participants lost to follow-up in the placebo group have survived, and had no serious adverse event.

We will present results of both scenarios in our review.

For all continuous outcome when analysing a 'beneficial outcome' will be the group mean plus two SDs (we will secondly use one SD in another sensitivity analysis) of the group mean and a 'harmful outcome' will be the group mean minus two SDs (we will seconds use one $\mathrm{SD}$ in another sensitivity analysis) of the group mean. ${ }^{103}$

To assess the potential impact of missing SDs for continuous outcomes, we will perform the following sensitivity analysis.

- Where SDs are missing and it is not possible to calculate them, we will impute SDs from trials with similar populations and low risk of bias. If we find no such trials, we will impute SDs from trials with a similar population. As the final option, we will impute SDs from all trials.

We will present results of this scenario in our review. Other post hoc sensitivity analyses might be warranted if unexpected clinical or statistical heterogeneity is identified during the analysis of the review results. ${ }^{103}$

\section{Summary of findings}

We will create a 'Summary of Findings' table using each of the primary outcomes (all-cause mortality, pain assessment on VAS or NRS, serious adverse event and quality of life). We will use the five GRADE considerations (bias risk of the trials, consistency of effect, imprecision, indirectness and publication bias) to assess the quality of a body of evidence as it relates to the studies which contribute data to the meta-analyses for the prespecified outcomes. ${ }^{103} 121-123$ We will use methods and recommendations described in Chapter 8 (Section 8.5) and Chapter 12 of the Cochrane Handbook for Systematic Reviews of Interventions ${ }^{85}$ using GRADEpro software. We will justify all decisions to downgrade the quality of studies using footnotes, and we will make comments to aid the reader's understanding of the review where necessary. First, we will present our results in the 'Summary of Findings' table based on the results from the trials with low risk of bias, and second, we will present the results based on all trials.

\section{Ethics and dissemination}

Ethical approval is not a requirement since no primary data will be collected. The findings of this systematic review will be submitted for peer-reviewed publication and disseminated in national and international conferences and is expected to inform healthcare workers and providers about the occurrence of serious and non-serious adverse events following cannabinoid consumption. It is expected that the findings of this systematic review will identify some research gaps for future trials.

\section{DISCUSSION}

This protocol aims at investigating the beneficial and harmful effects of cannabinoids in patients with any 
type of pain condition. The outcomes will be all-cause mortality, pain assessment on VAS or NRS, serious adverse events, quality of life, dependence, psychosis, non-serious adverse events and sleep quality.

This protocol has several strengths. The predefined methodology is based on the Cochrane Handbook for Systematic Reviews of Interventions, ${ }^{85}$ the eight-step assessment suggested by Jakobsen $e t$ al ${ }^{103}$ Trial Sequential Analysis ${ }^{84}$ and GRADE assessment. ${ }^{121-123}$ Hence, this protocol takes both the risk of random error and the risk of systematic error into account. We predefined evidence-based estimations of minimal important differences which will limit the risk of focusing on statistically significant results with questionable clinical importance. This threshold of minimal important difference is based on the estimations of several previously conducted studies and reviews. ${ }^{106} 107$ Moreover, we are including all types of cannabinoids and all types of pain which will increase the statistical power and make it possible to perform essential subgroup analyses. We have been in contact with several relevant patient associations which has assisted us in choosing the most clinically relevant outcomes.

Our protocol also has several limitations. One of the potential limitations is that we include participants with all types of pain; cannabinoids might have different effects on different types of pain. It might, for example, be problematic to combine trials assessing the effects of cannabinoids on acute pain and chronic pain because of different underlying pathophysiological mechanisms. ${ }^{124}$ On the other hand, the effects of cannabinoids on acute pain and chronic pain might be comparable and hence it might be valid to combine trials assessing the effects of cannabinoids on acute pain and chronic pain in meta-analysis, which would increase the statistical power. The results of the subgroup analysis comparing trials including participants with acute pain to participants with chronic pain will therefore be highlighted when reporting our review results. Moreover, we only intend to assess cannabinoids versus placebo or versus no intervention. Further systematic reviews with meta-analyses and Trial Sequential Analyses need to assess the benefits and harms of cannabinoids versus other pain killers, provided that cannabinoids show more benefit than harm in the present systematic review.

Furthermore, more than one active cannabinoid agent is often combined in the different intervention options provided to the patients with a pain condition, thereby making difficult to explore the analgesic effect and adverse event associated with a single cannabinoid agent. Hence, if we show a difference between the intervention options, it will be difficult to conclude what exactly caused the difference in effect. To minimise these limitations, we have planned a careful assessment of statistical and clinical heterogeneity as well as several subgroup analyses and sensitivity analyses. Another limitation is the large number of comparisons which increase the risk of type 1 error. We have adjusted our thresholds for significance according to the number of primary outcomes, but, as mentioned, we have also included multiple subgroup analyses. This large risk of type 1 error will be taken into account when interpreting the review results.

Acknowledgements We hugely appreciate the contribution of The Danish Diabetes Association, Steno Diabetes Centre Copenhagen, The Danish Rheumatism Association, The Danish Multiple Sclerosis Society and Danish Cancer Society in guiding us to select the most patient relevant outcomes. The expert help from Sarah Louise Klingenberg (Information Specialist, The Cochrane Hepato-Biliary Group, Copenhagen Trial Unit, Copenhagen, Denmark) in making the search strategy is hugely appreciated.

Contributors JB drafted the protocol. JCJ, SKK, OM, CG, JRF and MM amended the protocol. All authors read and approved the final manuscript.

Funding The authors have not declared a specific grant for this research from any funding agency in the public, commercial or not-for-profit sectors.

Competing interests None declared.

Patient consent for publication Not required.

Provenance and peer review Not commissioned; externally peer reviewed.

Open access This is an open access article distributed in accordance with the Creative Commons Attribution Non Commercial (CC BY-NC 4.0) license, which permits others to distribute, remix, adapt, build upon this work non-commercially, and license their derivative works on different terms, provided the original work is properly cited, appropriate credit is given, any changes made indicated, and the use is non-commercial. See: http://creativecommons.org/licenses/by-nc/4.0/.

\section{REFERENCES}

1 Verhaak PFM, Kerssens JJ, Dekker J, et al. Prevalence of chronic benign pain disorder among adults: a review of the literature. Pain 1998;77:231-9.

2 Kroenke K. Patients presenting with somatic complaints: epidemiology, psychiatric co-morbidity and management. Int $J$ Methods Psychiatr Res 2003;12:34-43.

3 Sternbach RA. Survey of pain in the United States: the Nuprin pain report. Clin J Pain 1986;2:49-53.

4 Gureje O, Von Korff M, Simon G, et al. Persistent pain and wellbeing: a world Health organization study in primary care. Jama 1998;280:147-51.

5 Breivik $\mathrm{H}$. International association for the study of pain: update on WHO-IASP activities. J Pain Symptom Manage 2002;24:97-101.

6 Astin J. Why patients use alternative medicine: results of a national study. JAMA 1998;279:1548-53.

7 Davison SN, Jhangri GS, Johnson JA. Cross-Sectional validity of a modified Edmonton symptom assessment system in dialysis patients: a simple assessment of symptom burden. Kidney Int 2006;69:1621-5.

8 Davison SN, Jhangri GS, Johnson JA. Longitudinal validation of a modified Edmonton symptom assessment system (ESAs) in haemodialysis patients. Nephrology Dialysis Transplantation 2006;21:3189-95.

9 Davison SN, Jhangri GS. Impact of Pain and Symptom Burden on the Health-Related Quality of Life of Hemodialysis Patients. J Pain Symptom Manage 2010;39:477-85.

10 Davison SN. Chronic pain in end-stage renal disease. Adv Chronic Kidney Dis 2005;12:326-34.

11 Kimmel PL, Emont SL, Newmann JM, et al. Esrd patient quality of life: symptoms, spiritual beliefs, psychosocial factors, and ethnicity. Am J Kidney Dis 2003;42:713-21.

12 Leinau L, Murphy TE, Bradley E, et al. Relationship between conditions addressed by hemodialysis guidelines and non-ESRDspecific conditions affecting quality of life. CJASN 2009;4:572-8.

13 Weisbord SD, Carmody SS, Bruns FJ, et al. Symptom burden, quality of life, advance care planning and the potential value of palliative care in severely ill haemodialysis patients. Nephrology Dialysis Transplantation 2003;18:1345-52.

14 Weisbord SD, Fried LF, Arnold RM, et al. Prevalence, severity, and importance of physical and emotional symptoms in chronic hemodialysis patients. JASN 2005;16:2487-94.

15 Gamondi C, Galli N, Schönholzer C, et al. Frequency and severity of pain and symptom distress among patients with chronic kidney disease receiving dialysis. Swiss Med Wkly 2013;143.

16 Smith $\mathrm{BH}$, Elliott AM, Chambers WA, et al. The impact of chronic pain in the community. Fam Pract 2001;18:292-9. 
17 Merskey H, Lindblom U, Mumford JM, et al. Part III: Pain terms-a current list with definitions and notes on usage with definitions and notes on usage. In: Merskey H, Bogduk N, eds. Classification of chronic pain, IASP Task force on taxonomy. 2nd Edition. IASP Press, 1994: 209-14.

18 Vučković S, Srebro D, Vujović KS, et al. Cannabinoids and pain: new insights from old molecules. Front Pharmacol 2018;9:1259.

19 Carr DB, Goudas LC. Acute pain. The Lancet 1999;353:2051-8.

20 Ashburn MA, Staats PS. Management of chronic pain. The Lancet 1999;353:1865-9.

21 Kanner R, Management P. Pain management. JAMA 1986;256:2112-4.

22 Loeser JD, Melzack R. Pain: an overview. The Lancet 1999;353:1607-9.

23 Portenoy R, Dhingra L. Assessment of cancer pain 2017 (cited 18/04 2018).

24 Gregory J, McGowan L. An examination of the prevalence of acute pain for hospitalised adult patients: a systematic review. J Clin Nurs 2016;25:583-98.

25 Treede R. Entstehung der schmerzchronifizierung. Rückenschmerzen und nackenschmerzen. Berlin, Heidelberg: Springer, 2016.

26 American Geriatrics Society Panel on the Pharmacological Management of Persistent Pain in Older Persons. Pharmacological management of persistent pain in older persons. J Am Geriatr Soc 2009;57:1331-46.

27 Breivik H, Collett B, Ventafridda V, et al. Survey of chronic pain in Europe: prevalence, impact on daily life, and treatment. Eur J Pain 2006:10:287-333

28 Goldberg DS, McGee SJ. Pain as a global public health priority. BMC Public Health 2011;11:770.

29 Gureje O, Von Korff M, Kola L, et al. The relation between multiple pains and mental disorders: results from the world mental health surveys. Pain 2008:135:82-91.

30 Institute of Medicine (US) Committee. on Advancing Pain Research, Care, and Education, Relieving pain in America: A blueprint for transforming prevention, care, education, and research. National Academies Press, 2011.

31 Koleva D, Krulichova I, Bertolini G, et al. Pain in primary care: an Italian survey. Eur J Public Health 2005;15:475-9.

32 Mäntyselkä $P$, Kumpusalo $E$, Ahonen $R$, et al. Pain as a reason to visit the doctor: a study in Finnish primary health care. Pain 2001;89:175-80.

33 Treede R-D, Rief W, Barke A, et al. A classification of chronic pain for ICD-11. Pain 2015;156:1-7.

34 Kelly DJ, Ahmad M, Brull SJ. Preemptive analgesia I: physiological pathways and pharmacological modalities. Can J Anesth/J Can Anesth 2001;48:1000-10.

35 Pogatzki-Zahn EM, Segelcke D, Schug SA. Postoperative painfrom mechanisms to treatment. PAIN Reports 2017;2:e588.

36 Lipowski ZJ. Chronic idiopathic pain syndrome. Ann Med 1990;22:213-7.

37 Goucke CR. The management of persistent pain. Medical Journal of Australia 2003:178:444-7.

38 Chang V. Approach to symptom assessment in palliative care, 2018.

39 Knowles $\mathrm{CH}$, Aziz Q. Basic and clinical aspects of gastrointestinal pain. Pain 2009;141:191-209.

40 Stein SL, pain Cpelvic. Chronic pelvic pain. Gastroenterol Clin North Am 2013;42:785-800.

41 Schwartz ES, Gebhart GF. Visceral pain. Curr Top Behav Neurosci 2014;20:171-97.

42 Giamberardino M, Affaitati G, Costantini R. Chapter 24 referred pain from internal organs. Handb Clin Neurol 2006;81:343-61.

43 Jensen TS, Baron R, Haanpää M, et al. A new definition of neuropathic pain. Pain 2011;152:2204-5.

44 Headache Classification Committee of the International Headache Society. The International Classification of Headache Disorders, 3rd edition (beta version). Cephalalgia, 2013: 33. 629-808p.

45 Institute for clinical systems improvement. Health care guideline: assessment and management of chronic pain 2009.

46 United Nations office on drugs and crime. World drug report, United nations 2016

47 Russo E. Cannabinoids in the management of difficult to treat pain. Ther Clin Risk Manag 2008;4:245-59.

48 Ueda N, Tsuboi K, Uyama T. Metabolic enzymes for endocannabinoids and endocannabinoid-like mediators. Boston: Academic Press, 2015.

49 Devane WA, Hanus L, Breuer A, et al. Isolation and structure of a brain constituent that binds to the cannabinoid receptor. Science 1992:258:1946-9.
50 Mechoulam R, Ben-Shabat S, Hanus L, et al. Identification of an endogenous 2-monoglyceride, present in canine gut, that binds to cannabinoid receptors. Biochem Pharmacol 1995;50:83-90.

51 Sugiura T, Kondo S, Sukagawa A, et al. 2-Arachidonoylgylcerol: a possible endogenous cannabinoid receptor ligand in brain. Biochem Biophys Res Commun 1995;215:89-97.

52 Fisar Z. Phytocannabinoids and endocannabinoids. CDAR 2009;2:51-75

53 Häuser W, Fitzcharles M, Radbruch L, et al. Cannabinoids in pain management and palliative medicine. Deutsches Arzteblatt international 2017;114:627-34.

54 Hazekamp A, Ware MA, Muller-Vahl KR, et al. The medicinal use of cannabis and cannabinoids - An international crosssectional survey on administration forms. J Psychoactive Drugs 2013;45:199-210.

55 Watson SJ, Benson JA, Joy JE. Marijuana and medicine: assessing the science base: a summary of the 1999 Institute of medicine report. Arch Gen Psychiatry 2000;57:547-52.

56 Brenneisen R. Chemistry and analysis of phytocannabinoids and other cannabis constituents, in Marijuana and the Cannabinoids, EISohly M A, Editor. Totowa, NJ: Humana Press, 2007: 17-49.

57 Pertwee R. Cannabis and cannabinoids: pharmacology and rationale for clinical use. Pharmacy and Pharmacology Communications 1997:3:539-45.

58 Solinas M, Goldberg SR, Piomelli D. The endocannabinoid system in brain reward processes. Br J Pharmacol 2008;154:369-83.

59 Koppel BS, Brust JCM, Fife T, et al. Systematic review: efficacy and safety of medical marijuana in selected neurologic disorders: report of the Guideline Development Subcommittee of the American Academy of Neurology. Neurology 2014;82:1556-63.

60 Gorelick D, Saxon A, Hermann R. Cannabis use and disorder: Pathogenesis and pharmacology. UpToDate Cannabis use and disorder: Pathogenesis and pharmacology 2018.

61 Morean ME, Kong G, Camenga DR, et al. High school students use of electronic cigarettes to vaporize cannabis. Pediatrics 2015;136:611-6.

62 Loflin M, Earleywine M. No smoke, no fire: what the initial literature suggests regarding vapourized cannabis and respiratory risk. Can $J$ Respir Ther 2015;51:7-9.

63 Aviram J, Samuelly-Leichtag G. Efficacy of cannabis-based medicines for pain management: a systematic review and meta-analysis of randomized controlled trials. Pain Physician 2017;20:E755-96.

64 Lynch ME, Campbell F. Cannabinoids for treatment of chronic non-cancer pain; a systematic review of randomized trials. Br J Clin Pharmacol 2011;72:735-44.

65 Meng H, Johnston B, Englesakis M, et al. Selective cannabinoids for chronic neuropathic pain: a systematic review and metaanalysis. Anesth Analg 2017;125:1638-52.

66 Boychuk DG, Goddard G, Mauro G, et al. The effectiveness of cannabinoids in the management of chronic nonmalignant neuropathic pain: a systematic review. J Oral Facial Pain Headache 2015;29:7-14

67 Martín-Sánchez E, Furukawa TA, Taylor J, et al. Systematic review and meta-analysis of cannabis treatment for chronic pain. Pain Med 2009;10:1353-68.

68 Campbell FA, Tramèr MR, Carroll D. Are cannabinoids an effective and safe treatment option in the management of pain? A qualitative systematic review. BMJ 2001;323:13-16.

69 Deshpande A, Mailis-Gagnon A, Zoheiry N, et al. Efficacy and adverse effects of medical marijuana for chronic noncancer pain: systematic review of randomized controlled trials. Can Fam Physician 2015;61:e372-81.

70 Stevens AJ, Higgins MD. A systematic review of the analgesic efficacy of cannabinoid medications in the management of acute pain. Acta Anaesthesiol Scand 2017;61:268-80.

71 Walitt B, Klose P, Fitzcharles M-A, et al. Cannabinoids for fibromyalgia. Cochrane Database Syst Rev 2016;9.

72 Mücke M, Phillips T, Radbruch L, et al. Cannabis-Based medicines for chronic neuropathic pain in adults. Cochrane Database Syst Rev 2018;112.

73 Boydell J, Van Os J, Caspi A, et al. Trends in cannabis use prior to first presentation with schizophrenia, in south-east London between 1965 and 1999. Psychol Med 2006;36:1441-6.

74 Andréasson S, Engström A, Allebeck P, et al. Cannabis and schizophrenia a longitudinal study of Swedish conscripts. The Lancet 1987:330:1483-6.

75 Arseneault L, Cannon M, Poulton R. Cannabis use in adolescence and risk for adult psychosis: longitudinal prospective study. BMJ 2002:325:1212-3. 
76 van Os J, Bak M, Hanssen M. Cannabis use and psychosis: a longitudinal population-based study. Am J Epidemiol 2002;156:319-27.

77 Zammit S, Allebeck P, Andreasson S. Self reported cannabis use as a risk factor for schizophrenia in Swedish conscripts of 1969: historical cohort study. BMJ 2002;325:1199.

78 Fergusson DM, Horwood LJ, Ridder EM. Tests of causal linkages between cannabis use and psychotic symptoms. Addiction 2005;100:354-66.

79 Henquet C, Krabbendam L, Spauwen J, et al. Prospective cohort study of cannabis use, predisposition for psychosis, and psychotic symptoms in young people. BMJ 2005;330.

80 Di Forti M, Quattrone D, Freeman TP, et al. The contribution of cannabis use to variation in the incidence of psychotic disorder across Europe (EU-GEI): a multicentre case-control study. The Lancet Psychiatry 2019;6:427-36.

81 Shamseer L, Moher D, Clarke M, et al. Preferred reporting items for systematic review and meta-analysis protocols (PRISMA-P) 2015 elaboration and explanation. BMJ 2015;349:g7647.

82 Moher D, Shamseer L, Clarke M, et al. Preferred reporting items for systematic review and meta-analysis protocols (PRISMA-P) 2015 statement. Syst Rev 2015;4:1.

83 International Conference on harmonisation of technical requirements for registration of pharmaceuticals for human use $(\mathrm{ICH})$ adopts consolidated guideline on good clinical practice in the conduct of clinical trials on medicinal products for human use. Int Dig Health Legis 1997;48:231-4.

84 Richards JR, Bing ML, Moulin AK, et al. Cannabis use and acute coronary syndrome. Clinical toxicology (Philadelphia, Pa) 2019:1-11.

85 Higgins J, Green S. Cochrane handbook for systematic reviews of interventions version 5.1.0, 2011. Available: www.handbook. cochrane.org

86 The Cochrane Collaboration. Review manager (RevMan. Copenhagen: the Nordic Cochrane Centre, The Cochrane Collaboration, 2014.

87 TSA - Trial Sequential Analysis. Copenhagen trial unit. Available: http://www.ctu.dk/tsa/

88 StataCorp: Stata: Release 142014College Station, TXStataCorp LP

89 Moher D, Liberati A, Tetzlaff J, et al. Preferred reporting items for systematic reviews and meta-analyses: the PRISMA statement. PLoS Med 2009;6:e1000097.

90 Gluud LL. Bias in clinical intervention research. Am J Epidemiol 2006;163:493-501.

91 Kjaergard LL, Villumsen J, Gluud C. Reported methodologic quality and discrepancies between large and small randomized trials in meta-analyses. Ann Intern Med 2001;135:982-9.

92 Lundh A, Sismondo S, Lexchin J, et al. Industry sponsorship and research outcome. Cochrane Database Syst Rev 2012;12.

93 Moher D, Pham Ba', Jones A, et al. Does quality of reports of randomised trials affect estimates of intervention efficacy reported in meta-analyses? The Lancet 1998;352:609-13.

94 Schulz KF, Chalmers I, Hayes RJ, et al. Empirical evidence of bias. dimensions of methodological quality associated with estimates of treatment effects in controlled trials. JAMA 1995;273:408-12.

95 Wood L, Egger M, Gluud LL, et al. Empirical evidence of bias in treatment effect estimates in controlled trials with different interventions and outcomes: meta-epidemiological study. BMJ 2008;336:601-5.

96 Savović J, Jones HE, Altman DG, et al. Influence of reported study design characteristics on intervention effect estimates from randomised controlled trials: combined analysis of metaepidemiological studies. Health Technol Assess 2012;16:1-82.

97 Higgins JPT, Thompson SG. Quantifying heterogeneity in a metaanalysis. Stat Med 2002;21:1539-58.

98 Higgins JPT, Thompson SG, Deeks JJ. Measuring inconsistency in meta-analyses. BMJ 2003;327:557-60.

99 Harbord RM, Egger M, Sterne JAC. A modified test for small-study effects in meta-analyses of controlled trials with binary endpoints. Stat Med 2006;25:3443-57.

100 Egger M, Smith GD, Schneider M, et al. Bias in meta-analysis detected by a simple, graphical test. BMJ 1997;315:629-34.

101 Begg CB, Mazumdar M. Operating characteristics of a RANK correlation test for publication bias. Biometrics 1994;50:1088-101.
102 Elbourne DR, Altman DG, Higgins JPT, et al. Meta-Analyses involving cross-over trials: methodological issues. Int J Epidemiol 2002;31:140-9.

103 Jakobsen JC, Wetterslev J, Winkel P, et al. Thresholds for statistical and clinical significance in systematic reviews with meta-analytic methods. BMC Med Res Methodol 2014;14:120.

104 Hagg O, Fritzell P, Nordwall A. The clinical importance of changes in outcome scores after treatment for chronic low back pain. Eur Spine J 2003;12:12-20.

105 Jaeschke R, Singer J, Guyatt GH. Measurement of health status. ascertaining the minimal clinically important difference. Control Clin Trials 1989;10:407-15

106 Olsen MF, Bjerre E, Hansen MD, et al. Pain relief that matters to patients: systematic review of empirical studies assessing the minimum clinically important difference in acute pain. BMC Med 2017;15:35

107 Olsen MF, Bjerre E, Hansen MD, et al. Minimum clinically important differences in chronic pain vary considerably by baseline pain and methodological factors: systematic review of empirical studies. J Clin Epidemiol 2018;101:87-106.

108 Keus F, Wetterslev J. Gluud C, and van Laarhoven CJ, Evidence at a glance: error matrix approach for overviewing available evidence. BMC Med Res Methodol 2010;10.

109 DerSimonian R, Laird N. Meta-Analysis in clinical trials. Control Clin Trials 1986; 7:177-88.

110 Demets DL. Methods for combining randomized clinical trials: strengths and limitations. Stat Med 1987;6:341-8.

111 Jakobsen JC, Wetterslev J, Lange T, et al. Viewpoint: taking into account risks of random errors when analysing multiple outcomes in systematic reviews. Cochrane Database of Systematic Reviews 2016.

112 Wetterslev J, Thorlund K, Brok J, et al. Trial sequential analysis may establish when firm evidence is reached in cumulative metaanalysis. J Clin Epidemiol 2008;61:64-75.

113 Thorlund KWJ, Brok J, Imberger G, et al. User manual for trial sequential analysis (TSA) 2011.

114 Brok J, Thorlund K, Gluud C, et al. Trial sequential analysis reveals insufficient information size and potentially false positive results in many meta-analyses. J Clin Epidemiol 2008;61:763-9.

115 Brok J, Thorlund K, Wetterslev J, et al. Apparently conclusive metaanalyses may be inconclusive-Trial sequential analysis adjustment of random error risk due to repetitive testing of accumulating data in apparently conclusive neonatal meta-analyses. Int J Epidemiol 2009;38:287-98.

116 Thorlund K, Devereaux PJ, Wetterslev J, et al. Can trial sequential monitoring boundaries reduce spurious inferences from metaanalyses? Int J Epidemiol 2009;38:276-86.

117 Wetterslev J, Thorlund K, Brok J, et al. Estimating required information size by quantifying diversity in random-effects model meta-analyses. BMC Med Res Methodol 2009;9:86.

118 Thorlund K, Anema A, Mills E. Interpreting meta-analysis according to the adequacy of sample size. An example using isoniazid chemoprophylaxis for tuberculosis in purified protein derivative negative HIV-infected individuals. Clin Epidemiol 2010;2:57-66.

119 Imberger G, Gluud C, Boylan J, et al. Systematic reviews of anesthesiologic interventions reported as statistically significant: problems with power, precision, and type 1 error protection. Anesth Analg 2015;121:1611-22.

120 Imberger G, Thorlund K, Gluud C, et al. False-Positive findings in Cochrane meta-analyses with and without application of trial sequential analysis: an empirical review. BMJ Open 2016;6:e011890.

121 Guyatt GH, Oxman AD, Vist GE, et al. Grade: an emerging consensus on rating quality of evidence and strength of recommendations. BMJ 2008;336:924-6.

122 Guyatt GH, Oxman AD, Schünemann HJ, et al. Grade guidelines: a new series of articles in the Journal of clinical epidemiology. J Clin Epidemiol 2011;64:380-2.

123 Schünemann HJ, Best D, Vist G, et al. Letters, numbers, symbols and words: how to communicate grades of evidence and recommendations. CMAJ 2003;169:677-80.

124 Voscopoulos C, Lema M. When does acute pain become chronic? Br J Anaesth 2010;105:i69-85. 